\title{
Primary extranodal soft-tissue B-cell lymphoma with abundant immunoglobulin inclusions mimicking adult rhabdomyoma: a case report
}

\author{
Zeng-Shan Li, Pei-Feng Li, Zhe Wang, Gao-Sheng Huang*
}

\begin{abstract}
Introduction: Immunoglobulin inclusions are found in B-cell neoplasms as well as in crystal-storing histiocytosis associated with B-cell lymphoproliferative disorders. At times, the deposits may be so profound as to obscure the diagnosis and may even lead to misdiagnosis. We report one case of low-grade extranodal lymphoplasmacytic lymphoma with abundant immunoglobulin inclusions and emphasize the need for immunophenotyping and molecular assay to make the right decision in diagnosis. To the best of our knowledge, this is the first report of extranodal B-cell lymphoma with abundant intracellular immunoglobulin accumulation.
\end{abstract}

Case presentation: A 62-year-old Asian man from China presented with a 13-year history of a right shoulder mass with recent ongoing pain. A desmoplastic fibroma located in the posterior muscles of the neck was suggested by magnetic resonance imaging, and extended local excision was performed. A biopsy, however, revealed large, isolated rhabdoid cells in a diffuse pattern with mild atypia and eosinophilic cytoplasm. Clustered lymphoid cells were interspersed among these cells. The diagnosis was initially suggested to be adult rhabdomyoma. The final diagnosis of lymphoma was made after immunohistochemical, ultrastructural and molecular studies.

Conclusion: We emphasize this histopathologic and immunohistochemical finding because of the potential for confusion with other tumors or disorders, such as adult rhabdomyoma or crystal-storing histiocytosis.

\section{Introduction}

Well-developed immunoglobulin (Ig) inclusions, such as Russell or Dutcher bodies, are very important diagnostic clues for some B-cell lymphomas, but diffuse and abundant intracellular Ig inclusions are unusual. Another condition in which unusual intracytoplasmic Ig inclusions can be found is crystal-storing histiocytosis associated with B-cell lymphoproliferative disorders. All of these factors may lead to a diagnostic dilemma $[1,2]$. We describe one case of low-grade lymphoplasmacytic lymphoma with abundant Ig inclusions and emphasize the need for the use of immunophenotyping and molecular assays to make the right decision in diagnosis and to distinguish these unusual cases from those involving other tumors or disorders.

\footnotetext{
* Correspondence: lizsh72@fmmu.edu.cn; huanggs@fmmu.edu.cn Department of Pathology, State Key Laboratory of Tumor Biology, Xi-Jing Hospital, Xi'an, Shaanxi 710032, Peoples Republic of China
}

\section{Case presentation}

A 62-year-old Asian man from China with a 13-year history of an enlarging right shoulder mass and recent ongoing pain was admitted to our hospital. His cervical lymph nodes were not palpable, and a review of all other systems was negative. A chest radiograph was normal. A desmoplastic fibroma located in the posterior muscles of the neck was suspected on the basis of computed tomography (CT) and magnetic resonance imaging (MRI) (Figure 1a), and extended local excision was performed. An $8 \mathrm{~cm} \times 6 \mathrm{~cm}$ poorly defined, intramuscular, homogeneous solid mass (Figure 1b) was studied with histology, histochemistry, immunohistochemistry, ultrastructural studies and molecular genetics.

Characteristically, the excised tumor was composed of large, isolated rhabdoid cells in a diffuse pattern with mild atypia and eosinophilic cytoplasm (Figure 2a). These cells were interspersed with clustered lymphoid cells. The diagnosis was initially suspected to be adult rhabdomyoma, but the tumor cells were negative for

C Biomed Central

(c) $2011 \mathrm{Li}$ et al; licensee BioMed Central Ltd. This is an Open Access article distributed under the terms of the Creative Commons Attribution License (http://creativecommons.org/licenses/by/2.0), which permits unrestricted use, distribution, and reproduction in any medium, provided the original work is properly cited. 


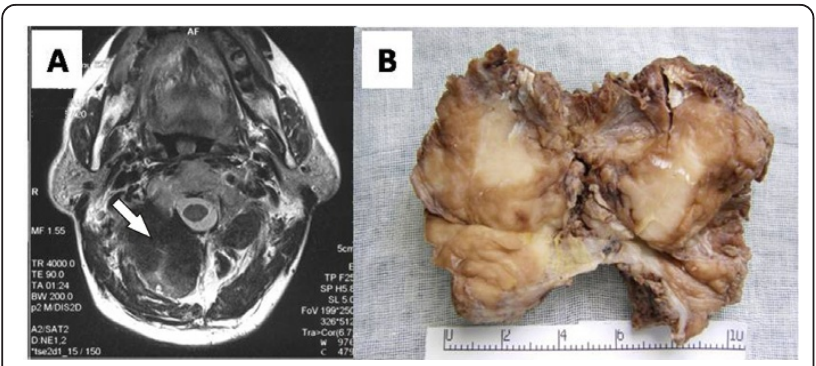

Figure 1 Views of the tissue mass. (a) Magnetic resonance imaging scan. A soft-tissue mass is seen in the right shoulder (white arrow). (b) Gross morphology.

immunostaining of desmin, muscle actin (HHF35), $\alpha$-smooth muscle actin ( $\alpha$-SMA) and myoglobin and positive for periodic acid-Schiff with diastase (PAS-D). Then a broad spectrum of immunohistochemical stains were used, and the tumor cells were immunoreactive for CD79 $\alpha$ (Figure 2b), CD20, CD43, paired box protein 5 (PAX5), leukocyte common antigen (LCA), and partially for CD5 (Additional file 1), but negative for CD138, CD68, CD163, CD3, synaptophysin and neuron-specific enolase. Ki-67 positivity was unevenly distributed in different areas, and the incidence was greater in the clustered lymphoid cells and almost zero in adjacent rhabdoid cell areas (Additional file 1). More interestingly, some of the eosinophilic inclusions were located in the nucleus, as demonstrated by periodic acid-Schiff stain (PAS)-PAX5 double staining (Figure 2c). Electron

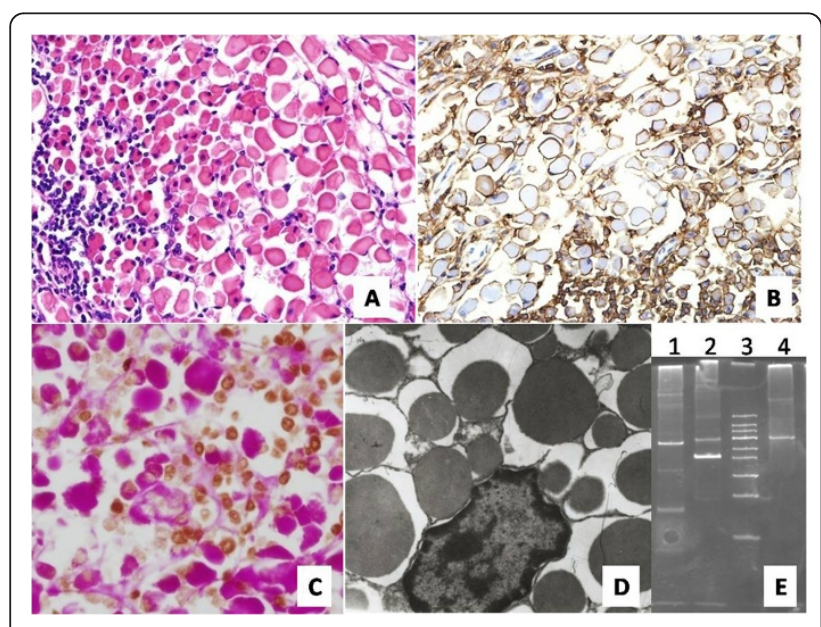

Figure 2 Results of studies. (a) Hematoxylin and eosin staining (original magnification, $\times 400$ ). (b) Immunohistochemical detection of CD79 $\alpha$ (original magnification, $\times 400$ ). (c) Double staining with periodic acid-Schiff stain (histochemistry) and paired box protein 5 (PAX5) (immunohistochemistry). (d) Electron microscopic micrograph of inclusion (original magnification, $\times 7,250$ ). (e) Lane 1 is immunoglobulin heavy chain $(\mathrm{IGH})$ rearrangements, lane 4 is immunoglobulin light chain kappa (IGK) rearrangements and lane 3 is a $50 \mathrm{bp}$ ladder. microscopy revealed pools of homogeneous, spherical, electron-dense bodies located in significantly dilated rough endoplasmic reticulum as well as extracellularly (Figure 2d). The mitochondria and lysosomes were poorly developed. Furthermore, monoclonality of the tumor cells was demonstrated by the presence of immunoglobulin heavy chain (IGH)-specific and immunoglobulin light chain kappa (IGK)-specific rearrangements (Figure 2e).

Other postoperative examinations showed that levels of IgE were elevated $(332 \mathrm{IU} / \mathrm{mL})$ and levels of IgA, IgM and IgG were normal. Bence-Jones protein was not found in the patient's urine. The final histopathologic diagnosis was consistent with a low-grade lymphoplasmacytic lymphoma with abundant Ig inclusions.

\section{Discussion}

Igs are reliable markers for the recognition of $\mathrm{B}$ lymphocytes and their neoplasms. In B-cell neoplasms, monotypic Ig is usually detected [3]. Igs may be located in the surface membrane, rough endoplasmic reticulum and perinuclear space. Well-developed Ig inclusions may appear as Russell or Dutcher bodies and give the strongest clue for the diagnosis. The Ig inclusions may be found in a range of B-cell neoplasms, including chronic lymphocytic leukemia, marginal zone B-cell lymphoma and follicular center cell lymphoma [4]. These inclusions are usually large, loosely packed fibrillar material not surrounded by a membrane or by rough endoplasmic reticulum, rodlike crystalline structures and, more rarely, signet ring-like vacuoles filled with microvesicles [5]. At times, the deposits may be so profound as to obscure the diagnosis and may even lead to misdiagnosis. In addition to lymphoma, unusual intracytoplasmic Ig inclusions can be found in crystal-storing histiocytosis associated with B-cell lymphoproliferative disorders such as diffuse large B-cell lymphoma, myeloma, lymphoplasmacytic lymphoma, marginal zone lymphoma and even non-neoplastic plasma cell proliferation $[1,2]$. It is a rare phenomenon in which macrophages accumulate lightchain or Ig crystalline inclusions [6]. All of these factors emphasize the need for immunophenotyping to make the right decision in diagnosis and distinguish these unusual cases from other tumors or disorders [2].

The case reported here was initially considered rhabdomyoma because of the morphologic features, the patient's age and the location of the tumor. The initial diagnosis was ruled out by immunohistochemical analysis that showed that tumor cells were negative for desmin, HHF35, $\alpha$-SMA and myoglobin and positive for PAS-D. Then we checked the slides carefully and found some Russell body-like intercellular eosinophilic bodies, which have the same characteristics as those in the cytoplasm, as well as the transition from small lymphoplasmacytic 
cells to those unique, large rhabdoid cells. Some other associated diseases were included in the differential diagnosis, including crystal-storing histiocytosis, extramedullary myeloma, and other Ig deposition diseases $[1,7]$. Finally, a special low-grade lymphoplasmacytic lymphoma with abundant intracytoplasmic Ig inclusions was demonstrated on the basis of immunohistochemical, ultrastructural and molecular studies. To the best of our knowledge, this is a rare case of primary malignant lymphoma with abundant intracytoplasmic Ig inclusions, as well as Dutcher bodies. The inclusions may be an outcome of impaired cell secretory activity, leading to Igs accumulation. It also may be associated with the excess production of a monoclonal Ig. It is not unusual that Bcell-associated disorders contain intracellular or intranuclear inclusion bodies such as Russell bodies, Dutcher bodies and other crystalline structures. Most of them are minor and not so evident compared with what we found in this case.

\section{Conclusion}

We report a rare case of low-grade B-cell lymphoma with abundant intracellular Ig accumulation. The patient's clinical presentation and MRI suggested a softtissue tumor, and histopathologic studies showed special rhabdoid cells with interspersed clustered lymphoid cells, thus providing a diagnostic dilemma. The final diagnosis was made with immunohistochemical, ultrastructural and molecular studies. It is important for pathologists to be aware of this rare extranodal Ig-rich lymphoma because of the potential for confusion with other tumors, such as rhabdomyoma and crystal-storing histiocytosis.

\section{Consent}

Written informed consent was obtained from the patient for publication of this case report and accompanying images. A copy of the written consent is available for review by the Editor-in-Chief of the journal.

\section{Additional material}

Additional file 1: Supplementary immunohistochemical results. Immunohistochemical detection of LCA, PAX-5, CD5, CD20, CD43 and Ki67 (original magnification, $\times 400$ ).

\section{Authors' contributions}

ZSL and PFL contributed equally to this work as first authors by providing data collection, data analysis and interpretation and manuscript writing. ZW and GSH contributed to the conception and design of the report. All authors read and approved the final manuscript.

\section{Competing interests}

The authors declare that they have no competing interests.
Received: 24 January 2010 Accepted: 7 February 2011

Published: 7 February 2011

\section{References}

1. Jones D, Bhatia VK, Krausz T, Pinkus GS: Crystal-storing histiocytosis: a disorder occurring in plasmacytic tumors expressing immunoglobulin kappa light chain. Hum Pathol 1999, 30:1441-1448.

2. Thorson P, Hess JL: Transformation of monocytoid B-cell lymphoma to large cell lymphoma associated with crystal-storing histiocytes. Arch Pathol Lab Med 2000, 124:460-462.

3. Mimura R, Hansmann ML, Lennert K: Immunoelectron microscopic localization of immunoglobulin in B-cell lymphomas. Virchows Arch B Cell Pathol Ind Mol Pathol 1986, 52:207-220.

4. Guglielmi P, Preud'Homme JL, Gourdin MF, Reyes F, Daniel MT: Unusual intracytoplasmic immunoglobulin inclusions in chronic lymphocytic leukaemia. Br J Haematol 1982, 50:123-134.

5. Van den Tweel JG, Taylor CR, Parker JW, Lukes RJ: Immunoglobulin inclusions in non-Hodgkin's lymphomas. Am J Clin Pathol 1978, 69:306-313.

6. Fairweather PM, Williamson R, Tsikleas G: Pulmonary extranodal marginal zone lymphoma with massive crystal storing histiocytosis. Am J Surg Pathol 2006, 30:262-267.

7. Suarez P, el-Naggar AK, Batsakis JG: Intracellular crystalline deposits in lymphoplasmacellular disorders. Ann Otol Rhinol Laryngol 1997, 106:170-172.

doi:10.1186/1752-1947-5-53

Cite this article as: Li et al:: Primary extranodal soft-tissue B-cell lymphoma with abundant immunoglobulin inclusions mimicking adult rhabdomyoma: a case report. Journal of Medical Case Reports 2011 5:53.

\section{Submit your next manuscript to BioMed Central and take full advantage of:}

- Convenient online submission

- Thorough peer review

- No space constraints or color figure charges

- Immediate publication on acceptance

- Inclusion in PubMed, CAS, Scopus and Google Scholar

- Research which is freely available for redistribution

Submit your manuscript at www.biomedcentral.com/submit
C Biomed Central 